\title{
SOME CHARACTERISTICS OF CHOICE BEHAVIOR IN RISKY SITUATIONS*
}

\author{
C. H. Coombs and D. G. Pruitt† \\ University of Michigan, Ann Arbor, Mich.
}

It has been observed that, before deciding between alternatives, people often enumerate the possible outcomes of choosing each alternative. They give each outcome a value representing its desirability, and often form some sort of estimate of the probability of occurrence of each outcome. Scientists have long believed that it should be possible to predict the direction of decision on the basis of these estimates of the value and probability of outcomes. Several models for making such predictions have been proposed (for example, Bernoulli, 1954; Edwards, 1955) based on the concept that people choose the alternative with the greatest expectation (the sum over outcomes of the probability times the value of each outcome). These models are weak because they can be made to predict such a wide range of behavior that it is difficult to design a crucial experiment. This weakness has led us to seek new directions for model building.

Two concepts upon which such a revision might be based are suggested in the current literature, both concerning elements of risk in alternatives: (1) "probability preferences," the concept that people are more attracted to certain probabilities of winning or losing than to others (Edwards, 1953); and (2) "variance preferences," the concept that people prefer certain dispersions of values of possible outcomes (Fisher, 1906; Allais, 1953). The present experiment was designed to find out whether probability and variance preferences exist and to discover any interactions that may occur between them. In addition, a model of variance preferences was tested that made more specific predictions than the expected utility interpretation (Bernoulli, 1954), a version of the expected value model.

An earlier report on this study has already been published (Coombs and Pruitt, 1960). In the present paper, the model for preferential choice behavior will be reviewed and the earlier report summarized. Then some additional analyses will be presented concerning inconsistency and transitivity of preference, group scales, and sex differences in probability and variance preferences.

\section{A Model for Variance Preferences}

The model is based upon a theory of preferential choice and the unfolding technique discussed in detail elsewhere (Coombs, 1952). Consider a set of alternatives, each having two outcomes at probabilities $p$ and $q$ respectively and all alternatives having the same expected value. The amount of variance of each alternative may be considered a point on a line or continuum of vari-

* The work described in this paper was supported in part by a research grant from the National Science Foundation, Washington, D.C., and by Project Michigan, a project of the University of Michigan in the field of Combat Surveillance. This project was sponsored by the Department of the Army, Washington, D.C., and was administered by the Signal Corps, U.S. Army, under Contract DA-36-039 ac 78801 .

$\dagger$ Postdoctorate Research Fellow, Public Health Service, Bethesda, Md. Present address: Northwestern University, Evanston, Ill. 
ance.* Assuming that an individual has an ideal variance, corresponding to a point on this same continuum, and that he prefers, of any two variances, the one nearer his ideal variance, then his preference ordering of the alternatives is the rank order of the alternatives obtained by folding this scale at his ideal point: the alternatives being ranked in order of increasing absolute distance from his ideal point. It will be convenient to speak of the variance scale generically as a joint scale or $J$ scale (both stimulus points and the ideal points of individuals are located on it); and the preference ordering of an individual, which is a folded $J$ scale, will be called an $I$ scale.

Consider the midpoints between stimuli on the $J$ scale. The order of the midpoints from left to right on the $J$ scale is determined in part by the relative distances between the stimuli. For example, if the distance between the first two stimuli, $A$ and $B$, is greater than the distance between the next two stimuli, $\mathrm{C}$ and $\mathrm{D}$, that is, if $\overline{\mathrm{AB}}>\overline{\mathrm{CD}}$, then the midpoint of stimuli $\mathrm{A}$ and $\mathrm{D}$ precedes the midpoint of stimuli $\mathrm{B}$ and $\mathrm{C}$. The $J$ scale is divided into segments that are bounded by the midpoints. To each such segment there corresponds a unique ordering of the absolute distances of the stimuli from any point in the segment. This ordering is, by hypothesis, the preference ordering of an individual whose ideal point lies in that segment.

It is evident that while the stimuli may be in the same rank order on the $J$ scale for different individuals, the relative distances between the stimuli may not be the same if the measures of the stimuli are subjective rather than objective. A consequence of this is that the order of the midpoints may be different for different individuals, and the sets of preference orderings corresponding to the different orders of midpoints will not be completely identical. It is convenient, therefore, to make the following distinction: if the order of the midpoints appears to be the same for all individuals, the $J$ scale is called a "quantitative $J$ scale" (that is, at least a common ordered-metric scale), whereas if the midpoint order is not the same but only the rank order of the stimuli, the $J$ scale is called a "qualitative $J$ scale" (that is, at most, a common ordinal scale).

In summary, a preference ordering of an individual, called an $I$ scale, is regarded as a folded $J$ scale. If a set of preference orderings, obtained from a number of individuals, may be unfolded to construct a $J$ scale with no contradictory order relations on intervals between stimuli, the $J$ scale is called a quantitative $J$ scale. If the set of $I$ scales may be unfolded to construct a $J$ scale on which only the order of the stimuli is common, the $J$ scale is called a qualitative $J$ scale.

\section{METHOD}

The stimuli were two-outcome bets, such as the following: $1 / 3$ chance to win 50 cents, and $2 / 3$ chance to lose 25 cents. All bets had an expected value of zero. The bets were organized into five sets. Sets I, II, and III each contained 6 bets that differed in variance but had the same probability of winning. In set $\mathrm{I}, \mathrm{P}=1 / 3$; in set II, $\mathrm{P}=1 / 2$; and in set III, $\mathrm{P}=2 / 3$. The same six

${ }^{*}$ In constructing the stimuli of this experiment, variance was defined as $V=p q(a-b)^{2}$. However, any measure of dispersion is admissible under the model as a definition of variance. 
variance levels were used in all three sets: $\$ 0.12,0.30,1.00,5.00,25.00$, and 100.00. Sets IV and V each contained 5 bets that differed in probability of winning but had the same variance. The variance of set IV was $\$ 1.00$; of set $\mathrm{V}, \$ 25.00$. The five differing probabilities were $1 / 6,1 / 3,1 / 2,2 / 3$ and 5/6. Sets I, II, and III were used to study variance preferences under constant probability treatment; sets IV and $\mathbf{V}$ were used to study probability preferences under constant variance treatment. Within each set the bets were exhaustively paired, and each pair was presented 8 times.

The bets were given to the subjects in dittoed booklets constructed so that one replication on all pairs was completed before the next was started. The $S$ s were told to choose, in each pair, the bet they "would prefer to play" and to indicate this decision by making a check in a box beside the bets. Ninetynine students at the University of Michigan served as subjects.

\section{Summary of Findings Previousiy Reported}

\section{Consistency and Transitivily of Preference}

An information measure was used to eliminate the few $S$ s in each set who were essentially random in their choices. This measure provided a convenient index number to characterize an individual's degree of inconsistency of choice in each set of stimuli. Comparison of these indices across sets of stimuli revealed that individual differences in inconsistency are statistically significant and moderate in magnitude.

Weak stochastic transitivity was tested and found to be satisfied for most individuals. When this condition is satisfied, the bets can be simply ordered from left to right for each individual so that each bet is preferred at least one half the time to any bet to the right of it. This is taken to be his preference ordering or $I$ scale.

\section{Unfolding Analysis of Preferences}

Variance preferences. The preference orderings ( $I$ scales) for each set of stimuli were unfolded, and a best quantitative $J$ scale was constructed. In all three sets (I, II, and III), the vast majority of preference orderings fit the same quantitative $J$ scale, and most of those that did not fit deviated in minor ways, apparently as a result of inconsistency. Thus the results gave strong support to the proposed model of variance preferences. However, it was not possible to answer with assurance the question whether a common quantitative or only a common qualitative $J$ scale of variance underlies choice. Although the findings seem to support the former conclusion, this might have resulted from the choice of stimuli.

Probability preferences. Again the preference orderings were unfolded for each set. However, in this case, only about 60 per cent of the cases in each set fitled the same quantitative $J$ scale, a scale of increasing probability of winning. In most of the preference orderings that did not fit, the trouble seemed to lie with bets at $1 / 2$ chance of winning, which were too much preferred. When these bets were eliminated from the analysis, a much larger proportion of the cases fitted the same quantitative $J$ scale of increasing probability of winning. 


\section{Stability and Interaction of Preference Patterns}

For almost every individual, the probability preference patterns at the two levels of variance (sets IV and V) were remarkably similar, suggesting that probability preferences are stable in the face of changing variance. On the other hand, an individual's variance preferences were often quite different at different probability treatments (sets I, II, and III). These differences were largely a function of probability preferences: at probability levels that people preferred (as shown by their behavior in sets IV and V) they preferred more variance.

TABLE 1

Distribution of $\overrightarrow{\mathrm{H}}$ Within Stimulus Sets

\begin{tabular}{c|c|c|c|c|c}
\hline & \multicolumn{5}{|c|}{ Stimulus sets* } \\
\cline { 2 - 6 } & \multicolumn{3}{|c|}{ Variance preferences } & Probability preferences \\
\cline { 2 - 7 } & I & II & III & IV & V \\
\hline $0-0.1000$ & 15 & 37 & 21 & 6 & 7 \\
$0.1001-0.2000$ & 14 & 15 & 14 & 14 & 13 \\
$0.2001-0.3000$ & 14 & 16 & 9 & 18 & 7 \\
$0.3001-0.4000$ & 14 & 12 & 13 & 7 & 18 \\
$0.4001-0.5000$ & 13 & 9 & 10 & 12 & 11 \\
$0.5001-0.6000$ & 10 & 3 & 10 & 20 & 13 \\
$0.6001-0.7000$ & 12 & 3 & 10 & 6 & 15 \\
$0.7001-0.8000$ & 1 & 1 & 5 & 8 & 10 \\
$0.8001-0.9000$ & 4 & 1 & 3 & 5 & 4 \\
$0.9001-1.000$ & 2 & 2 & 4 & 3 & 1 \\
$\overline{\mathbf{H}} \ddagger$ & 0.368 & 0.229 & 0.367 & 0.434 & 0.443 \\
\hline
\end{tabular}

* The entries in all but the bottom row refer to number of $S \mathbf{s}$.

$\dagger$ Higher $\overrightarrow{\mathrm{H}}$ indicates greater inconsistency.

$\ddagger \overline{\overline{\mathrm{H}}}$ is a mean $\overline{\mathrm{H}}$.

\section{Additional Analyses}

\section{Inconsistency of Preference}

The obtained distribution of the information measure of inconsistency, $\overline{\mathrm{H}}$, is given in TABLE 1. The average degree of inconsistency for each of the stimulus sets is contained in the bottom row of the table, indicating that probability preferences were most inconsistent, variance preferences at $P$ 's of $1 / 3$ and $2 / 3$ next most inconsistent, and variance preferences at $1 / 2$ most consistent.

In a previous study of inconsistency of preferences (Coombs, 1958) it was shown that laterality of stimuli on the $J$ scale relative to an individual's ideal affected the order of magnitude of his inconsistency. For individuals toward the extreme of a $J$ scale the pairs of stimuli are predominantly unilateral, and for individuals with intermediate ideals, there are more bilateral pairs. Variability in the location of an individual's ideal inflates the inconsistency of bilateral judgments but has no effect on unilateral judgments. Since all individuals are assumed to have variable ideals, one would expect the inconsistency measure of an individual's preference to be higher the nearer he is to the middle 
of the $J$ scale. These conclusions are borne out by the results presented in TABLE 2. Stimulus sets I, II, and III have 6 stimuli each, designated A to $\mathrm{F}$ in order of increasing variance, in this table. Sets IV and $\mathrm{V}$ have only 5 stimuli, and the symbols $\mathrm{A}$ to $\mathrm{E}$ correspond to the probabilities $1 / 6$ to $5 / 6$ respectively. It is evident that, for each set of stimuli, inconsistency is greatest for individuals centrally located on the $J$ scale and diminishes toward either end.

TABLE 2

RELATION OF INCONSISTENCY TO IDEAL

\begin{tabular}{|c|c|c|c|c|c|c|c|c|c|c|c|c|c|c|c|}
\hline & \multicolumn{15}{|c|}{ Stimulus sets } \\
\hline & \multicolumn{3}{|c|}{ I } & \multicolumn{3}{|c|}{ II } & \multicolumn{3}{|c|}{ III } & \multicolumn{3}{|c|}{ IV } & \multicolumn{3}{|c|}{ V } \\
\hline & $\overline{\overline{\mathrm{H}}}^{*}$ & Md $\overline{\mathbf{H}} t$ & & $\overline{\overline{\mathbf{H}}}$ & $\mathrm{Md} \overline{\mathrm{H}}$ & $\mathrm{N}$ & $\overline{\mathbf{H}}$ & $\mathrm{Md} \overrightarrow{\mathrm{H}}$ & $\mathrm{N}$ & $\overrightarrow{\overline{\mathbf{H}}}$ & $\mathrm{Md} \overline{\mathbf{H}}$ & $\mathbf{N}$ & $\overline{\overline{\mathbf{H}}}$ & $\mathbf{M d} \overline{\mathbf{H}}$ & $N$ \\
\hline $\begin{array}{l}\text { Most } \\
\text { preferred } \\
\text { stimulus }\end{array}$ & & & & & & & & & & & & & & & \\
\hline A & 0.2157 & $|0.1630|$ & 34 & 0.1802 & 0.1450 & 18 & 0.2582 & 0.1948 & 16 & 0.4070 & 0.3442 & 33 & 0.4566 & 0.4895 & 31 \\
\hline B & 0.4045 & $|0.3959|$ & 4 & 0.2167 & 0.2189 & 4 & 0.3500 & 0.3841 & 13 & 0.6252 & 0.5807 & 3 & 0.6884 & $\mid \begin{array}{l}0.7401 \\
0.5705\end{array}$ & 4 \\
\hline $\begin{array}{l}C \\
D\end{array}$ & $\begin{array}{l}0.4520 \\
0.4884\end{array}$ & $\mid \begin{array}{l}0.4979 \\
0.4824\end{array}$ & $\begin{array}{l}16 \\
12\end{array}$ & $\begin{array}{l}0.2846 \\
0.4138\end{array}$ & $\left|\begin{array}{l}0.2657 \\
0.4273\end{array}\right|$ & $\begin{array}{l}11 \\
12\end{array}$ & $\begin{array}{l}0.5689 \\
0.5188\end{array}$ & $\begin{array}{l}0.5743 \\
0.5452\end{array}$ & $\begin{array}{r}8 \\
14\end{array}$ & $\begin{array}{l}0.5231 \\
0.4288\end{array}$ & $\begin{array}{l}0.5173 \\
0.4341\end{array}$ & $\begin{array}{r}21 \\
9\end{array}$ & $\begin{array}{l}0.5366 \\
0.4512\end{array}$ & $\begin{array}{r}0.5295 \\
0.4053\end{array} \mid$ & $\begin{array}{r}15 \\
6\end{array}$ \\
\hline $\mathrm{E}$ & $\begin{array}{l}0.484 \\
0.4586\end{array}$ & $\left|\begin{array}{l}0.4824 \\
0.4471\end{array}\right|$ & $\begin{array}{r}12 \\
9\end{array}$ & 0.2547 & $\mid \begin{array}{l}0.2627 \\
0 .\end{array}$ & 15 & 0.3626 & $|0.3472|$ & 5 & 0.2978 & 0.3253 & 29 & 0.3341 & 0.3397 & 38 \\
\hline $\mathrm{F}$ & 0.2112 & $|0.2169|$ & 17 & 0.1095 & $|0.0362|$ & 36 & 0.2359 & $|0.1812|$ & $37 \mid$ & & & & & & \\
\hline
\end{tabular}

$* \overline{\overline{\mathrm{H}}}$ is the mean $\overline{\mathrm{H}}$.

$\dagger \overrightarrow{\mathbf{H}}$ indicates inconsistency.

$\ddagger N$ indicates number of $S$ s.

\section{Strong Stochastic Transitivity}

Whether inconsistent preferential choices satisfy strong stochastic transitivity is of some interest. Strong stochastic transitivity (s.s.t.) is defined as follows for any triple $(a, b, c)$ :

$$
\mathrm{P}(a, b) \geqslant 0.5 \text { and } \mathrm{P}(b, c) \geqslant 0.5<=\mathrm{P}(a, c) \geqslant \max \{\mathrm{P}(a, b), \mathrm{P}(b, c)\}
$$

It is clear that data satisfying this condition would also satisfy the condition for weak stochastic transitivity. The distinction is made clear in this example. Suppose that $\mathrm{P}(a, b)=0.60$ and $\mathrm{P}(b, c)=0.70$. If $\mathrm{P}(a, c)$ is at least 0.50 then weak stochastic transitivity is satisfied by the ordering $a b c$. Strong stochastic transitivity requires that $\mathrm{P}(a, c)$ be at least 0.70 . It is obvious that a monotone transformation of inconsistency in to psychological distance, as in the Thurstone (1927) and Luce (1959) models, requires strong stochastic transitivity.

While no simple statistical test exists for testing whether the data of this experiment satisfy strong stochastic transitivity, a simple tabulation of the degree to which they do is of interest. This tabulation is presented in TABLE 3. Strong stochastic transitivity is satisfied by only a small number of those subjects whose preference orderings satisfy weak stochastic transitivity (column $b$ ). It must be kept in mind that each pairwise probability is based on only eight observations and it is to be expected that even if strong stochastic transi- 
tivity held in principle, a certain number of $S$ s would fail to satisfy it. Unfortunately no statistical test may readily be made of whether the observed number is a significant departure: one only forms the impression that this may be so.

Further evidence is contained in the number of triples that fail to satisfy strong stochastic transitivity. In stimulus sets I, II, and III, there are 20 triples imbedded in the weak stochastic ordering of the 6 stimuli in each set. In sets IV and V, with 5 stimuli, there are 10 triples in each. The failure of an individual's ordering to satisfy strong stochastic transitivity is due to the failure of one or more of these triples. Column $c$ of TABLE 3 contains the total number of triples, in each stimulus set, multiplied by the number of $S$ s. Column $d$ gives the number of such triples that fail to satisfy strong stochastic transitivity and column $e$ indicates the proportion. It is apparent that, on the average, about 25 per cent of all triples fail to satisfy strong stochastic

TABLE 3

Number of $S \mathrm{~s}$ and Triples Satisfying Strong Stochastic Transitivity

\begin{tabular}{c|c|c|c|c|c|c}
\hline Stimulus set & $a$ & \multicolumn{1}{c|}{$b$} & $c$ & $d$ & $e$ & $f$ \\
\hline II & 86 & 9 & 1720 & 442 & 0.26 & 5.74 \\
II & 91 & 31 & 1820 & 308 & 0.17 & 5.13 \\
III & 85 & 14 & 1700 & 453 & 0.27 & 6.38 \\
IV & 89 & 18 & 890 & 219 & 0.25 & 3.08 \\
V & 88 & 13 & 880 & 243 & 0.28 & 3.24 \\
\hline
\end{tabular}

(a) Total number of $S \mathbf{s}$ satisfying weak stochastic transitivity.

(b) Total number of $S$ s satisfying strong stochastic transitivity.

(c) Total number of triples in the weak stochastic orderings.

(d) Total number of triples failing to satisfy strong stochastic transitivity.

(e) Proportion of triples failing to satisfy strong stochastic transitivity.

(f) Mean number of triples failing to satisfy strong stochastic transitivity for those $S_{\mathrm{s}}$ who depart from strong stochastic transitivity.

transitivity. For those $S \mathrm{~s}$ who fail to satisfy strong stochastic transitivity, the average number of triples in their rank order that violate this condition is given in the last column of the table. This indicates that failure to satisfy strong stochastic transitivity is not a consequence of just one or two triples but is perhaps a significant departure.

Considerations of the laterality of stimuli on the $J$ scale relative to an individual's ideal (Coombs, 1958) also suggest that strong stochastic transitivity would be less often satisfied by people whose ideals are nearer the middle of the distribution. Rather than present all the results in detail a summary is contained in TABLE 4. All variance preference $I$ scales are divided into three groups, those beginning with one of the end stimuli ( $\mathrm{A}$ or $\mathrm{F}$ ) and those beginning with an intermediate stimulus. The number of such preference scales and the number of triples in their preference scales that satisfy strong stochastic transitivity are presented in TABLE 4 . As is evident, the expectation is borne out. The results on probability preferences show the same trend, but not as strongly, probably due to the fact that there are fewer stimuli (and hence less effect of laterality), and because of the strong preference for $P=1 / 2$. 


\section{Group Preferences}

Preference scales based on the pooled data for all individuals in a stimulus set are of some interest. A group preference scale may be constructed by majority choice on each pair if weak stochastic transitivity holds for the group, with each individual getting one vote on each pair. The result is a rank order that is a maximum likelihood preference scale for the entire group. The results are presented in TABLE 5 .

Each of these group scales may be seen to correspond to an $I$ scale and the corresponding $I$ scale number may be obtained by counting the number of

TABLE 4

Number of Subjects and Triples Satisfying Strong Stochastic Transitivity Related to Ideai

\begin{tabular}{c|c|c|c|c|c|c}
\hline & $a$ & $b$ & $c$ & $d$ & $e$ & $f$ \\
\hline A & 64 & 14 & 0.219 & 1280 & 1035 & 0.809 \\
B to $\mathrm{E}$ & 106 & 7 & 0.066 & 2120 & 1513 & 0.714 \\
F & 83 & 32 & 0.386 & 1660 & 1361 & 0.820 \\
\hline
\end{tabular}

(a) Number of $I$ scales.

(b) Number of $S$ s satisfying strong stochastic transitivity.

(c) Proportion of $S$ s satisfying strong stochastic transitivity.

(d) Total number of triples.

(e) Number of triples satisfying strong stochastic transitivity.

(f) Proportion of triples satisfying strong stochastic transitivity.

TABLE 5

Group Preference Scales

\begin{tabular}{c|c|c}
\hline & $\begin{array}{c}\text { Stochastically-dominant group } \\
\text { preference scale }\end{array}$ & $\begin{array}{c}\text { Corresponding } I \text { scale } \\
\text { number }\end{array}$ \\
\hline Probability treatment & CBADEF & 4 \\
$\mathrm{P}=1 / 3$ & EDCBAF & 11 \\
$\mathrm{P}=1 / 2$ & DECBAF & 10 \\
$\mathrm{P}=2 / 3$ & 32415 & 5 \\
Variance treatment & 34521 & 8 \\
Low & & \\
High & & \\
\hline
\end{tabular}

midpoints crossed to obtain the group scale and adding one. These numbers are given in the last column of the table. It is evident that, as a group, maximum variance is preferred at $50-50$ odds and more variance is preferred at favorable than at unfavorable odds. At higher variance the group tends to prefer odds more in their favor, although the prior analysis of variance (Coombs and Pruitt, 1960) suggests that this is not a significant difference. In all cases the group scales are folded $J$ scales and are different under different treatments.

Another way of characterizing the preferences of a group is through the distribution of the ideals (first choices) of its members. Such distributions are shown in the histograms of FIGUREs 1 and 2. In all cases it will be seen that the distributions are U-shaped, with roughly one third of the subjects favoring each of the two extreme stimuli and one third favoring the intermediate stimuli. 
A method frequently used for obtaining a group scale is to determine the total number of times each stimulus has been preferred in its pairwise presentation with all others. This vote-count method for preferential choice has been shown to be not independent of other alternatives (Coombs, 1958) when preferences are generated from a joint scale, and it has not been used here.
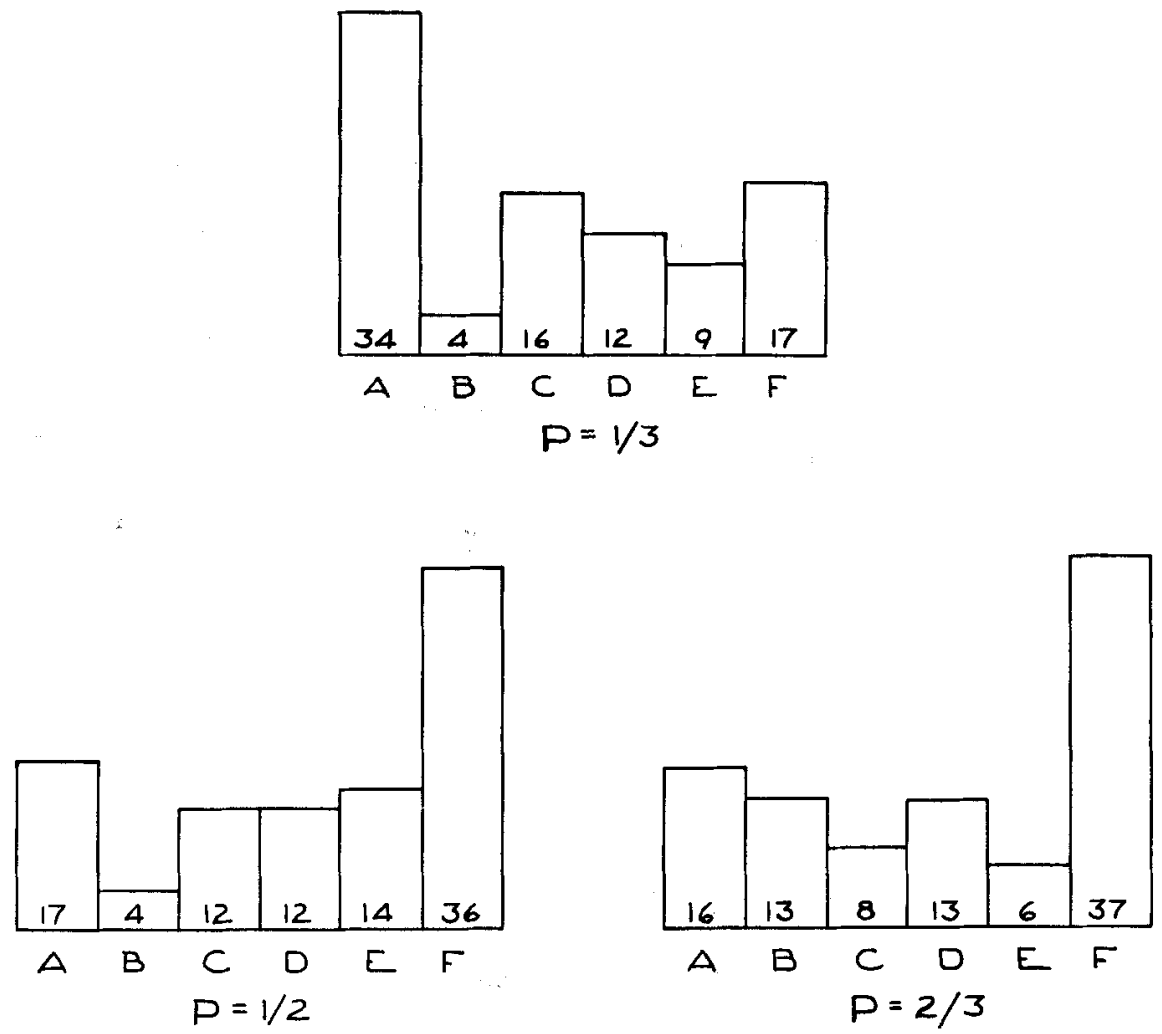

FIGURE 1. Frequency of first choices in variance preferences.
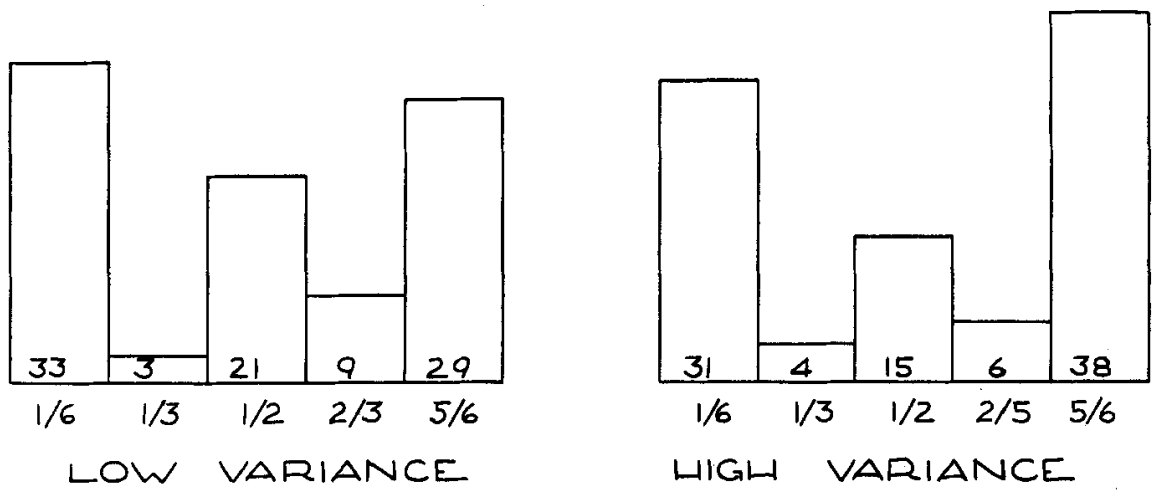

Figure 2. Frequency of first choices in probability preferences. 


\section{Sex Differences}

Inconsistency. In TABLE 6 is shown the mean $\overline{\mathrm{H}}$ for men and women separately on variance and probability preferences, and TABLE 7 contains an analysis of variance. It is clear that the men are more consistent than the women in their preference behavior in the case of both variance and probability preferences. There is no significant interaction between sex and treatments in the case of either variance or probability preferences.

Transitivily. These differences are not worth reporting in detail. There are slightly more intransitive $I$ scales, and $I$ scales with ties, for women than for men, but these may be attributed to the greater inconsistency of the women.

Preference. A distribution of the first choices of men and women in their variance preferences reveals a significant tendency for women to fold the $J$

TABle 6

Mean $\overrightarrow{\mathrm{H}}$ for Variance and Probability Preferences of Men and Women

\begin{tabular}{|c|c|c|c|c|c|}
\hline \multirow{2}{*}{ Sex } & \multicolumn{5}{|c|}{ Stimulus sets } \\
\hline & I & II & III & IV & $\mathrm{v}$ \\
\hline $\begin{array}{l}\text { Male } \\
\text { Female }\end{array}$ & $\begin{array}{l}0.3203 \\
0.4025\end{array}$ & $\begin{array}{l}0.1902 \\
0.2570\end{array}$ & $\begin{array}{l}0.3114 \\
0.4083\end{array}$ & $\begin{array}{l}0.3834 \\
0.4708\end{array}$ & $\begin{array}{l}0.3803 \\
0.4893\end{array}$ \\
\hline
\end{tabular}

TABLE 7

Test of Sex Differences in Inconsistency of Variance and Probability Preferences

\begin{tabular}{|c|c|c|c|c|c|c|}
\hline \multirow{2}{*}{ Source } & \multicolumn{3}{|c|}{ Variance preferences } & \multicolumn{3}{|c|}{ Probability preferences } \\
\hline & $d f$ & $M S$ & $F$ & $d f$ & $M S$ & $F$ \\
\hline $\begin{array}{l}\text { Sexes } \\
\text { Error }\end{array}$ & $\begin{array}{r}1 \\
97\end{array}$ & $\begin{array}{l}0.4872 \\
0.1191\end{array}$ & $4.091^{*}$ & $\begin{array}{r}1 \\
97\end{array}$ & $\begin{array}{l}0.4561 \\
0.0926\end{array}$ & $4.925^{*}$ \\
\hline
\end{tabular}

Note: interaction of sexes and probability treatments not significant by inspection.

* Significant beyond the 0.05 level.

scale of variance, whereas men are more evenly distributed. This is borne out in the 3 fourfold tables presented in TABLE 8 .

The probability preferences also present a trend in sex differences, and the relevant data are contained in TABLE 9. Women tend to prefer "fair" bets and positively-skewed bets more than men, whereas the men seem to prefer the negatively-skewed bets more: these are the "almost sure things" with small probability of a large loss.

\section{Conclusions}

Findings on variance preferences give strong support to a model which asserts that, at each probability of winning, the individual has an ideal level of variance and will choose, of any two levels of variance, that which is closer to his ideal. A similar model for probability (skewness) preferences appears to hold, except that $50-50$ bets are excessively preferred. 
A vast proportion of the $S \mathrm{~s}$ were significantly consistent in their preferences and exhibited weak stochastic transitivity. On the other hand, strong stochastic transitivity was not met in most cases. Inconsistency of preferences and failure to satisfy strong stochastic transitivity were observed to be greater for individuals whose ideals are intermediate on the $J$ scale, compared with those whose ideals are extreme. This is to be expected from the effect of laterality on the inconsistency of preferences.

TABLE 8

Sex Differences in Variance Premerences

\begin{tabular}{|c|c|c|c|c|c|c|}
\hline & \multicolumn{6}{|c|}{ Stimulus sets } \\
\hline & \multicolumn{2}{|c|}{ I } & \multicolumn{2}{|c|}{ II } & \multicolumn{2}{|c|}{ III } \\
\hline & Male & Female & Male & Female & Male & Female \\
\hline $\begin{array}{l}\text { Var. prefs. } \\
\text { Extreme } \\
\text { Folded }\end{array}$ & $\begin{array}{l}27 \\
13\end{array}$ & $\begin{array}{l}24 \\
28\end{array}$ & $\begin{array}{l}28 \\
13\end{array}$ & $\begin{array}{l}25 \\
30\end{array}$ & $\begin{array}{l}26 \\
13\end{array}$ & $\begin{array}{l}28 \\
26\end{array}$ \\
\hline Test & \multicolumn{2}{|c|}{$\begin{array}{l}x^{2}=4.125 \\
1 d f \stackrel{P}{\mathrm{P}}<0.05\end{array}$} & \multicolumn{2}{|c|}{ 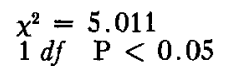 } & \multicolumn{2}{|c|}{ n.s. } \\
\hline
\end{tabular}

TABLE 9

Sex Differences in Probability Preferences

\begin{tabular}{|c|c|c|c|c|}
\hline & \multicolumn{4}{|c|}{ Stimulus sets } \\
\hline & \multicolumn{2}{|c|}{ IV: Low variance } & \multicolumn{2}{|c|}{ V: High variance } \\
\hline & Male & Female & Male & Female \\
\hline $\begin{array}{l}1 / 6 \text { and } 1 / 3 \\
1 / 2 \\
2 / 3 \text { and } 5 / 6\end{array}$ & $\begin{array}{r}16 \\
4 \\
22\end{array}$ & $\begin{array}{l}20 \\
17 \\
16\end{array}$ & $\begin{array}{r}13 \\
2 \\
26\end{array}$ & $\begin{array}{l}22 \\
13 \\
18\end{array}$ \\
\hline Test & \multicolumn{2}{|c|}{$\chi^{2}=\underset{\mathrm{P}}{6}<0.71,2 d f$} & \multicolumn{2}{|c|}{$\begin{array}{c}\chi^{2}=8.51 \\
P\end{array}$} \\
\hline
\end{tabular}

Group scales based on majority choice were folded $J$ scales, and they revealed that for the group as a whole, maximum variance was preferred at odds of one half, and more variance was preferred at favorable than at unfavorable odds; at the higher variance level the group tended to prefer odds in their favor. Distributions of first-choice were U-shaped for all sets of stimuli.

Women differed from men in that they (1) were more inconsistent, (2) were correspondingly less often transitive, (3) preferred intermediate variance more often than men, and (4) preferred even money and long shot bets more than men. It is not clear whether women's greater inconsistency is due to the laterality effect, in that their ideals tend to be intermediate, or whether they may be said to be intrinsically more inconsistent in this kind of behavior. 


\section{ACKNOWLEDGMENTS}

We express our appreciation for many helpful comments and criticisms from Patrick Suppes and Jack Sawyer, who are not be be held responsible for the use we made of their suggestions.

\section{REFERENCES}

Allais, M. 1953. Le comportement de l'homme rationnel devant le risque. Critique des postulates et axioms de l'école Américaine. Econometrika. 21: 503-546.

BernoulLi, D. 1954. Exposition of a new theory on the measurement of risk. Econometrika. 22: 23-36.

Coomss, C. H. 1952. A theory of psychological scaling. Engr. Research Bull. No. 34. Univ. Mich. Press. Ann Arbor, Mich.

CoomBs, C. H. 1958. On the use of inconsistency of preference in psychological measurement. J. Exptl. Psychol. 65: 1-7.

Coombs, C. H. \& D. G. Pruitr. Components of risk in decision making: Probability and variance preferences. J. Exptl. Psychol. In press.

Edwards, W. 1953. Probability preferences in gambling. Am. J. Psychol. 66: 349-364.

EDwards, W. 1955. The prediction of decisions among bets. J. Exptl. Psychol. 60: 201214.

FIsher, I. 1906. The Nature of Capital and Income. Macmillan. New York, N. Y.

LUCE, R. D. 1959. Individual Choice Behavior: A Theoretical Analysis. Wiley. New York, N. Y.

Thurstone, L. L. 1927. A law of comparative judgment. Psychol. Rev. 34: 273-286. 